\title{
Magnetic dipole moment generated in nano-droplets irradiated by circularly polarized laser pulse
}

\author{
Zs. Lécz $\left(^{*}\right.$ \\ ELI-ALPS, ELI-HU NKft. Dugonics square 13., 6720 Szeged, Hungary
}

A. Andreev (1)

Max Born Institute for Nonlinear Optics and Short Pulse Spectroscopy, 12489 Berlin, Germany and ELI-ALPS, ELI-HU NKft. Dugonics square 13., 6720 Szeged, Hungary

(Received 20 January 2020; accepted 3 April 2020; published 28 April 2020)

\begin{abstract}
A new mechanism to generate nanometer-scale magnetic dipoles is presented with the help of particle-in-cell simulations and analytical modeling. The kilo-tesla axial magnetic field is sustained by electrons orbiting around spherical clusters after the interaction with a circularly polarized laser pulse. The magnetization of material in such way is different from the inverse Faraday rotation and the localized magnetic fields have a much higher amplitude.
\end{abstract}

DOI: 10.1103/PhysRevResearch.2.023088

\section{INTRODUCTION}

Since the discovery of electron's spin magnetic moment scientists have been interested in manipulating the quantized magnetic-dipole moment of elementary particles or atoms [1-3]. The material properties are strongly influenced by the alignment of well-separated magnetic fields of atoms or molecules. On the nanometer scale structured material surfaces provide a playground for studying tunable magnetic systems [4] which may help to understand basic phenomena related to dipole-dipole coupling [5,6] or other nontrivial interactions [7-9]. On the other hand, temporal variation of such exotic magnetic fields leads to magnetic-dipole radiation $[10,11]$, which is an uncommon source of electromagnetic waves in nature.

In the astrophysical community and in fundamental particle physics the magnetic-field amplitude is the most important quantity which has been pushed to the limit of hundreds of tesla in conventional nondestructive devices [12]. Higher magnetic field requires higher current density, which is achieved in $Z$-pinch experiments [13]. Recently, owing to the development of high power lasers, new $Z$-pinching methods have been investigated in nanowire array targets which could provide megatesla field amplitudes with micrometer scale lengths $[14,15]$. Huge toroidal or azimuthal magnetic fields are relatively easy to produce because they require longitudinal electric current, which is naturally produced by the light pressure of intense pulses [16-19]. Axial magnetic fields, which possess some features of a magnetic dipole,

\footnotetext{
*zsolt.lecz@eli-alps.hu

Published by the American Physical Society under the terms of the Creative Commons Attribution 4.0 International license. Further distribution of this work must maintain attribution to the author(s) and the published article's title, journal citation, and DOI.
}

are commonly produced by circularly polarized laser pulses via the effect of inverse Faraday rotation [20-22]. Special laser pulses with screw-shaped intensity distribution have been proposed for the generation of gigagauss axial magnetic field [23]. However, these methods provide nonstationary and short-living magnetic fields in underdense plasma. On the macroscopic level in a submillimeter spatial domain nanosecond-long pulses with $\mathrm{kJ}$ energy have been applied to generate kilotesla magnetic field with a capacitor-coil configuration [24,25].

In the present work we propose a method of generating large amplitude magnetic-dipole moment based on the electron inertia in clusterized (nano-droplet) rare-gas targets $[26,27]$ irradiated by circularly polarized ultrashort pulses. It is stable and stays almost constant on the timescale of modern ultrashort $(\sim 10 \mathrm{fs})$ laser pulses. In contrast with the uniform density underdense plasma in our method, the magnetic dipoles are well localized at the positions of the overdense droplets and their number is equal to the number of droplets inside the laser focal volume. The most unique feature of this nanoscale magnet is the toroidal current surrounding the droplet, which could open new possibilities in modern electrodynamics [28]. In the presented scheme laser pulses with $\sim \mathrm{mJ}$ energy are sufficient, therefore magnetization of material at $\mathrm{kHz}$ repetition rate is possible. The magnetic field decreases slowly after the laser-droplet interaction and we show that the decay rate is proportional to the laser electric field and inversely proportional to the droplet mass.

\section{PHYSICAL SETUP}

The simulation tool we use is the 3D EPOCH particle-in-cell code. We assume an already-ionized spherical target (nanodroplet) with radius $R=50 \mathrm{~nm}$. The ion charge state is $Z=$ 20 and the ions are Xe with number density $n_{0}=10^{28} \mathrm{~m}^{-3}$ [27,29]. The corresponding electron density in the target is $2 \times 10^{29} \mathrm{~m}^{-3}$ which is equivalent to $n_{e}=182 n_{c r}$ in the case 

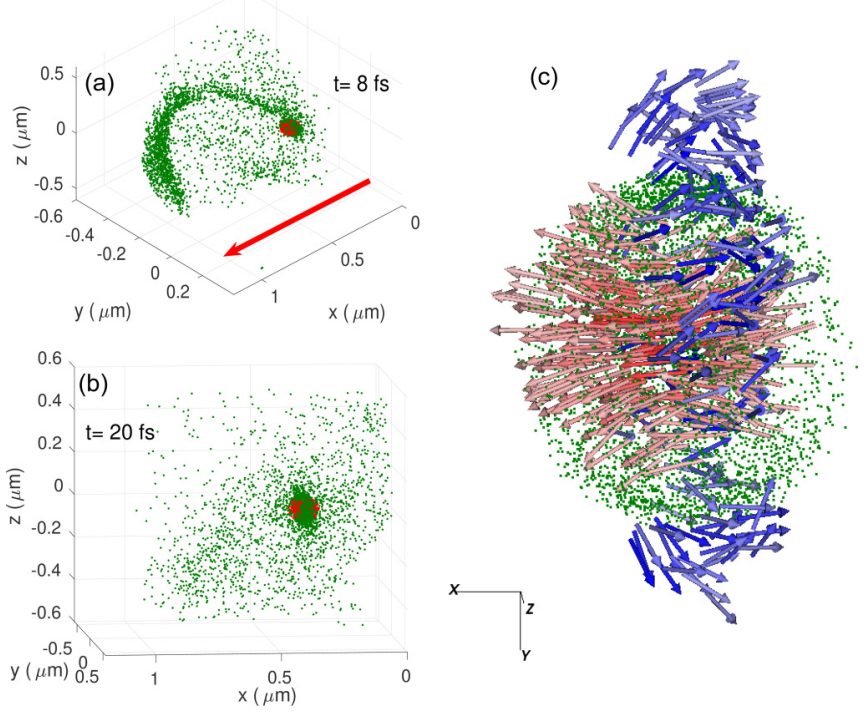

FIG. 1. (a) Spatial distribution of electrons (green) outside of the droplet during the interaction. The ions are shown by red dots. The red arrow indicates the direction of the laser pulse. (b) Distribution of particles after the interaction $\left(t_{L}=3 \mathrm{fs}\right.$ and the laser pulse leaves the target at $t=9 \mathrm{fs}$ ). (c) A zoom of the picture shown in panel (b). The arrows with red tones represent the magnetic field vectors with $100 T<B_{x}<1 k T$ and the arrows in blue correspond to $-100 T<$ $B_{x}<-30 T$.

of $\lambda_{L}=1 \mu \mathrm{m}$ radiation wavelength, where $n_{c r}=\omega_{L}^{2} m_{e} \epsilon_{0} / e^{2}$ is the critical density. The incoming laser pulse is a circularly polarized plane wave (the simulation domain is smaller than the laser focal spot size) with Gaussian temporal field profile, $I(t)=I_{L} \exp \left[-\left(t-t_{L}\right)^{2} / t_{L}^{2}\right]$. The peak intensity of the laser pulse is $I_{L}=4 \times 10^{18} \mathrm{~W} / \mathrm{cm}^{2}$ and its duration is varied in simulations.

The simulation domain has a volume of $1.5 \times 1 \times 1 \mu \mathrm{m}^{3}$ represented by $600 \times 400 \times 400$ grid cells and the target plasma is represented by 10 ion and 100 electron macroparticles per cell. At such high resolution one pseudo-electron contains only $\sim 10$ real electrons and the total number of macroparticles used to represent the target plasma is $10^{7}$. Since the radius of the electron trajectory becomes quickly much greater than the width of the simulation box we use absorbing boundaries for electrons and outgoing for electromagnetic waves. In a more realistic scenario one should use periodic boundary conditions in the transverse direction, because in experiments in the target there are many clusters, but for now we concentrate on a single nano-droplet. Later, larger simulation domains are also considered which contain more than 10 clusters and the distance between them is varied.

One representative case is shown in Fig. 1 with the parameters described above. Three major steps of the interaction are presented: Figure 1(a) shows the electron removal from the droplet, Fig. 1(b) shows the re-arrangement of the energetic electrons around the droplet, and in Fig. 1(c) the final stage is illustrated by showing the magnetic-field vectors inside (positive) and outside (negative) of the droplet. These three steps and the physics involved are described in great detail in the next section.

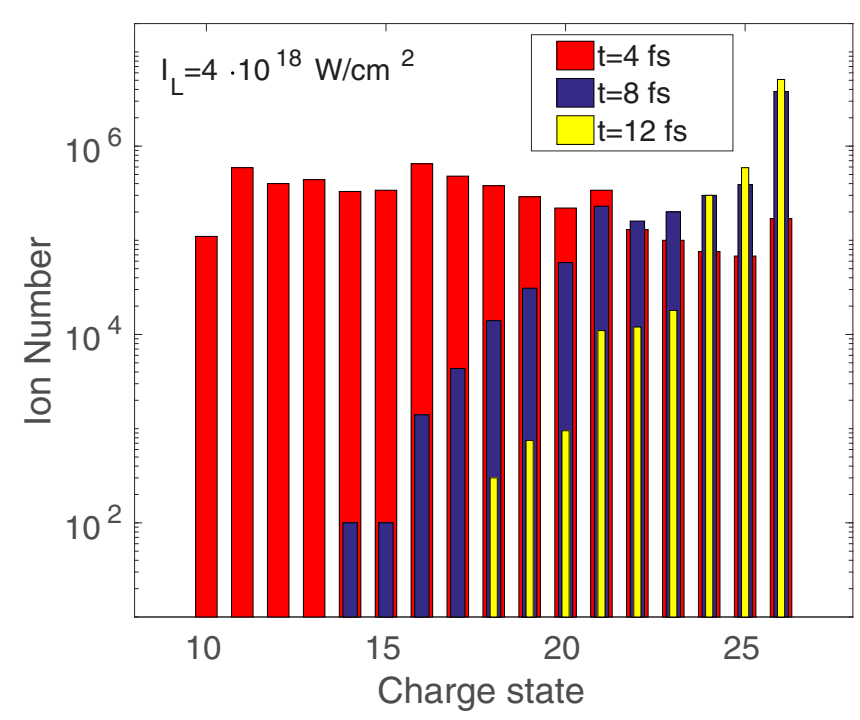

FIG. 2. Distribution of charge states at different time instances.

To check the average ionization state in the droplet and the effect of collisions we perform a simulation with an initially neutral Xe droplet interacting with the main pulse. Here we assume that the effect of prepulse is negligible, or it does not exist. In principle the prepulse can be absorbed by droplets close to the edge of the clusterized gas jet and the main pulse can interact with a fresh droplet somewhere in the middle of the target.

The distribution of different ion charges at early times is shown in Fig. 2. This simulation was done with the 3-fs-long (FWHM) pulse. One can see that, already at $8 \mathrm{fs}$, most of the ions have $26+$ charge, but later on the charge distribution changes slowly. The $27+$ level requires $1.5 \mathrm{keV}$ energy and it did not appear for these parameters. The magnetic field obtained in this simulation was very close to the one presented in Fig. 1, which indicates that a collisionless calculation is sufficient to investigate the process of magnetic-dipole generation.

In additional simulations we have considered already expanded droplets, as the effect of prepulse, and we observed only positive changes, since the field amplitude was much larger. This could be attributed to more efficient electron acceleration in the plasma corona, where resonance can take place. The preplasma greatly complicates the interaction. and its investigation is beyond of the scope of this paper, but it will be considered in future studies.

\section{RESULTS}

\section{A. Single droplet}

Before the stable state of the magnetic dipole there are two preceding steps already presented in Fig. 1 and examined in detail in Fig. 3 for a longer pulse. First, the laser field extracts a significant amount of electrons from the skin layer of the droplet and they are pushed away by the ponderomotive force. At the beginning of the interaction the electrons have a spiral path following the rotation of the electric field of the laser pulse and leave the target [Fig. 3(a)]. The azimuthal velocity $v_{\theta}$ of electrons appears due to the $B_{L x}$ component of the laser 

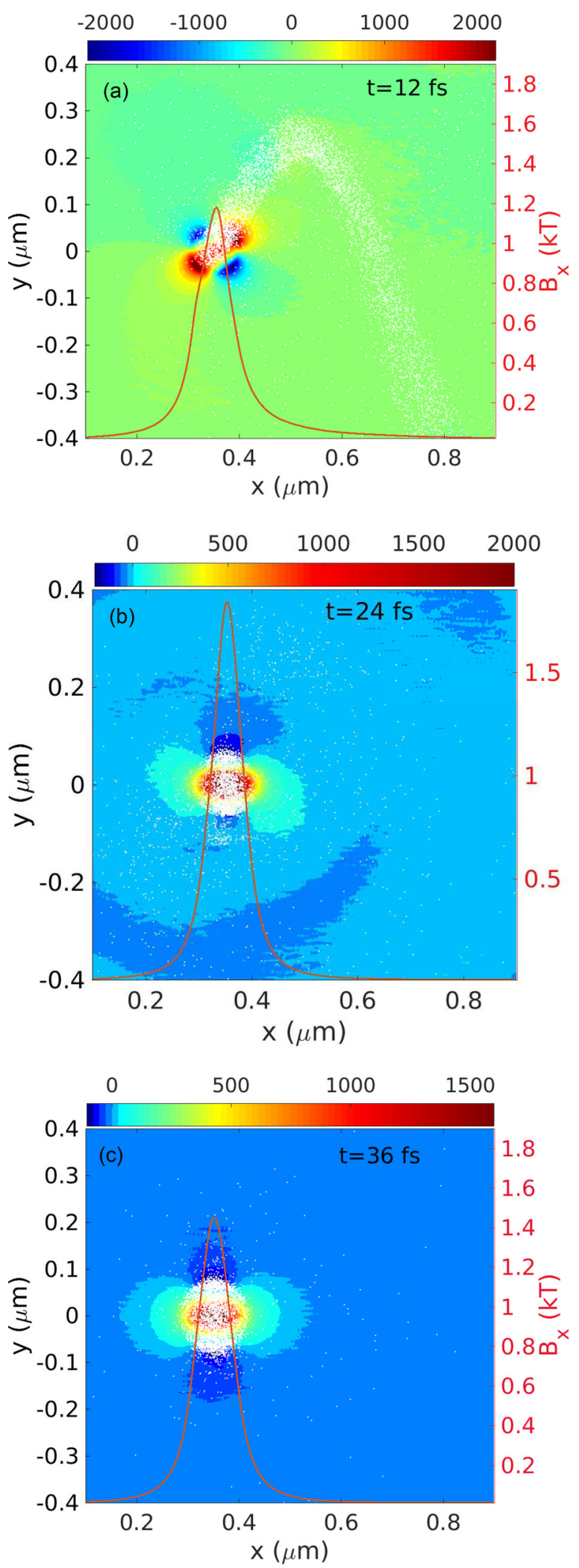

FIG. 3. Distribution of the $B_{x}$ (color bar in tesla) field in the $x y$ plane at different time instances in the simulation with $t_{L}=10 \mathrm{fs}$. The laser-droplet interaction ends at $t=22 \mathrm{fs}$. The white dots represent the position of electrons, which are outside of the droplet, projected to the $x y$ plane. The red lines show the axial lineout of the magnetic field. The laser peak intensity is $I_{L}=4 \times 10^{18} \mathrm{~W} / \mathrm{cm}^{2}$ $\left(a_{0}=1.7\right)$, it propagates from left to the right. field: $p_{\theta}=\gamma m_{e} v_{\theta}=e v_{r} B_{L x} t_{L}$, where $v_{r}$ is the radial velocity component. Thus, beside the longitudinal ponderomotive force there is an azimuthal driver of the motion.

Later on, the positive charge of the droplet weakens the extraction of electrons and keeps them near the target. In the second step, after the laser action, the electrons keep rotating due to the conservation of orbital angular momentum (OAM) and get attracted back by the highly charged droplet. This is a relaxation process, since the radial momentum is transformed into potential energy until all electrons are in equilibrium on a stable orbit in the electrostatic field of the droplet. The electrons can oscillate in the $x$ direction with an amplitude not greater than the Debye length, which is on the order of $100 \mathrm{~nm}$. The average longitudinal velocity is zero, therefore no azimuthal magnetic field is generated. In Fig. 3(b) the total charge is $2.3 \mathrm{pC}$ which is sufficient to force a large amount of electrons to a circular trajectory. Now the remaining radial velocity is transformed into $v_{\theta}$ by the self-generated magnetic field: $d p_{\theta} / d t=e v_{r} B_{x}$.

The nonthermal electrons, orbiting in the $y z$ plane, will possess only $v_{\theta}$ on average, which results in the azimuthal current responsible for the axial magnetic field. In Fig. 3(c) the magnetic-field distribution is axially symmetric and the rotation plane of these electrons is perpendicular to the laser axis, obeying the conservation law of OAM. One can see that the peak value of the magnetic field decreases in time which can be attributed to the expansion of the ion core, as shown later.

The laser field can act on the target electrons as long as the radial electric field is smaller than the laser field. When they become equal the maximum charge is reached, which can be expressed as

$$
Q_{m}=4 \pi \epsilon_{0} R^{2} E_{L} .
$$

For our target parameters one obtains $Q_{m}=2.4 \mathrm{pC}$, which is the absolute maximum charge reachable with the given droplet and laser pulse. The actual charge, which we denote as $Q_{*}$, is smaller in the case of ultrashort pulses and approaches asymptotically the value of $Q_{m}$ in the case of longer pulses. There is an upper limit of laser intensity at which all electrons are removed from the droplet. In the case of single-cycle pulses it is obtained from the equation $Q_{m}=4 \pi R^{3} n_{0} Z e / 3$, yielding $E_{L, \max }=R n_{0} Z e /\left(3 \epsilon_{0}\right)$, which for our parameters corresponds to the intensity of $I_{L, \max } \approx 10^{21} \mathrm{~W} / \mathrm{cm}^{2}$. For longer pulses the upper limit of intensity is lower, which is investigated later in this paper.

The temporal evolution of the charge buildup is crucial to understand the dynamics after the laser pulse leaves. In any kind of laser-solid interaction the laser field is canceled at the plasma-vacuum interface because of the generated surface current carried by the electrons. The corresponding electric field in the plasma skin layer can be calculated from the Ampere law: $E=e n_{e} c /\left(\epsilon_{0} \omega_{L}\right)=E_{L}$, where we assume that the electron velocity is close to the speed of light. From this equality one can easily find that the density of electrons displaced from the skin layer is $n_{e}=a_{0} n_{c r}$, where $a_{0}=$ $e E_{L} /\left(m_{e} \omega_{L} c\right)>1$ is the normalized laser electric field. In the case of finite-size target the transverse electric field generated by the target space charge has to be included as well, which leads to a lower effective electric field and to a different 

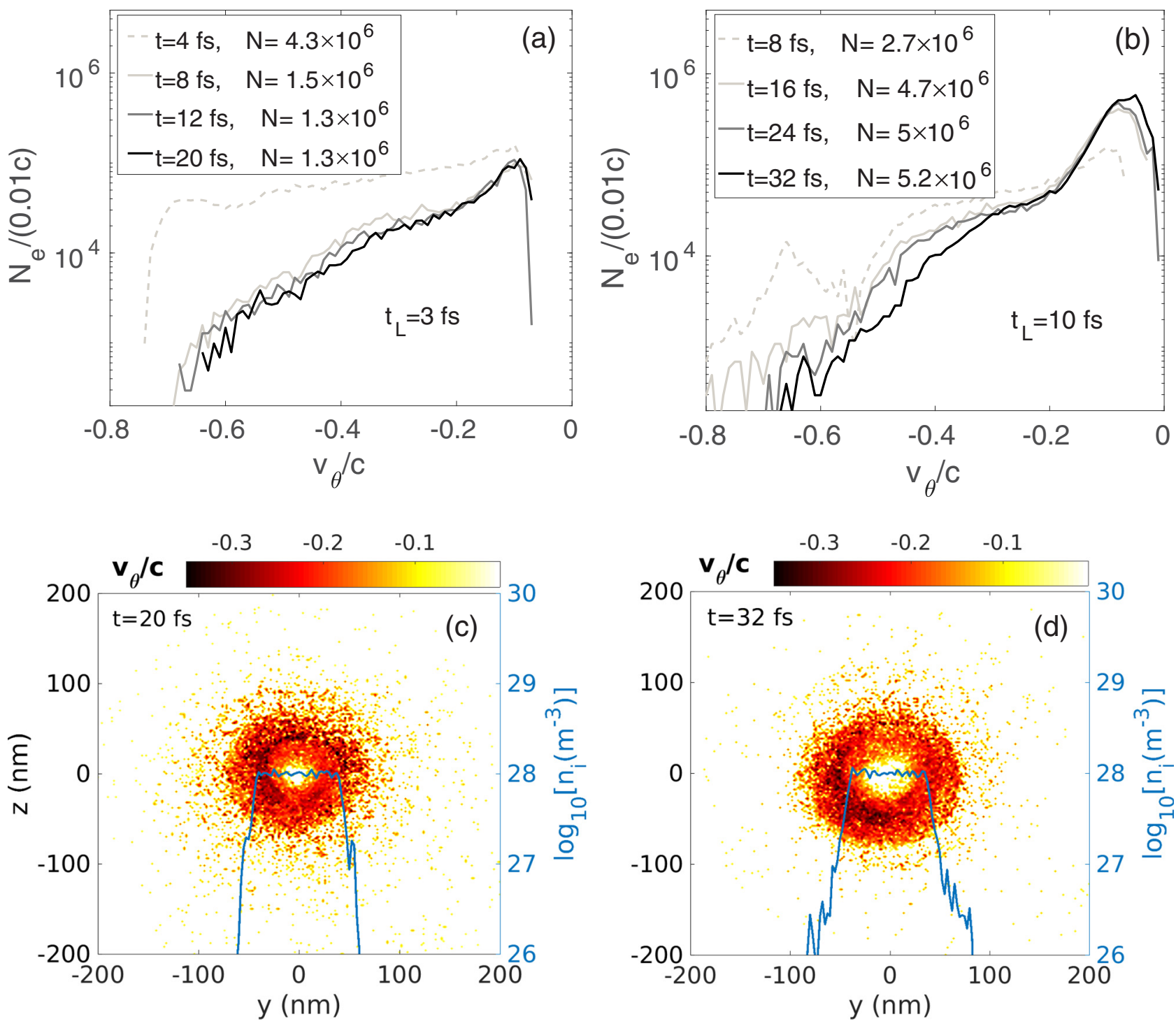

FIG. 4. (a), (b) Distribution of electrons which contribute to the azimuthal current in a limited spatial domain: $0.25 \mu \mathrm{m}<x<0.45 \mu \mathrm{m}$. The legend also shows the total number (integrated spectrum) of electrons, $N$. (c), (d) Distribution of average azimuthal velocity of electrons from the same spatial interval. Here electrons with $v_{\theta}<-0.2 c$ are included. The full lines show the ion density cross section of the expanded droplet.

electron density: $n_{e}^{\prime}=a n_{c r}$ with $a=e\left(E_{L}-E_{R}\right) /\left(m_{e} \omega_{L} c\right)$, where $E_{R}=Q /\left(4 \pi \epsilon_{0} R^{2}\right)$. For the analytical treatment we assume that the surface area of interaction is $S=\pi R^{2}$. Since the laser pulse has a relativistic intensity the electrons acquire a velocity close to the speed of light, which initially points mostly in the radial direction. The electron extraction during the laser pulse is continuous due to the circular polarization and the droplet charge can be described by the integral $Q=$ $\int e n_{e}^{\prime} S c d t$, or in form of a differential equation as

$$
\frac{d Q}{d t}=\frac{\omega_{L}}{4}\left(Q_{m}-Q\right)
$$

which can be solved exactly:

$$
Q(t)=Q_{m}\left[1-\exp \left(-\omega_{L} t / 4\right)\right] .
$$

In the simulation with $t_{L}=3$ fs the calculated droplet charge is $Q_{*}=Q\left(t_{L}\right)=1.8 \mathrm{pC}$, which is close to the value measured from the simulation $(1.7 \mathrm{pC})$. With a three-times-longer laser pulse, when $\omega_{L} t_{L} \gg 4$, the droplet charge reaches only $Q_{*}=2.3 \mathrm{pC}$, which is close to $Q_{m}$. This means that laser pulses much longer than a few cycles will not increase significantly the droplet charge but, as we show in the following, the rotational motion of the remaining electrons in the target can be significantly enhanced.

The distributions of the azimuthal electron velocity $\left(d N / d v_{\theta}\right)$ at different time instances are shown in Figs. 4(a) and 4(b) for two laser pulse durations. This velocity component is calculated from the orbital angular momentum as $v_{\theta}=L_{x} /\left(\gamma m_{e} r_{e}\right)$, where $L_{x}=\left(\mathbf{r}_{\mathbf{e}} \times \mathbf{p}\right)_{x}$ and $r_{e}$ is the radius of electron trajectory, measured from the center of the droplet. In these pictures only nonthermal electrons are included, which is achieved by calculating the difference between the electron spectrum with negative and positive $v_{\theta}$. The number of electrons $(N)$ contributing to the magnetic field is then obtained by integrating the spectrum. This number is only a few percent of the initial electron number present in the 

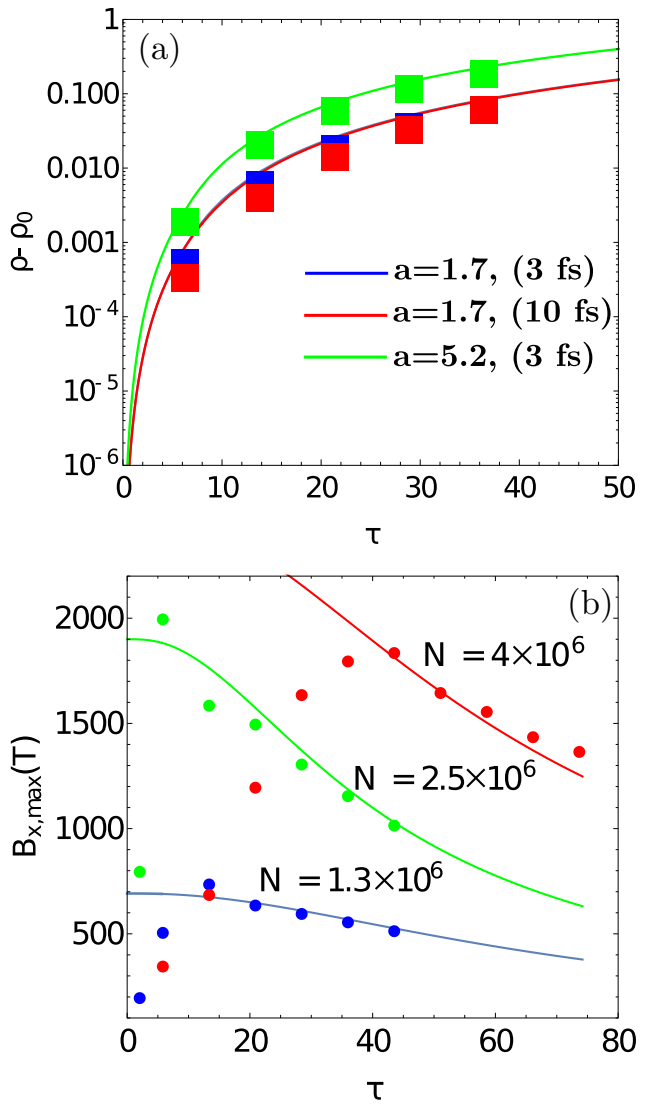

FIG. 5. (a) Temporal dependence of droplet radius from three different simulations compared with the results of Eq. (6). (b) Time evolution of the peak magnetic field for the same simulations compared with Eq. (4). Here $N$ is a fitting parameter, which is in good agreement with the measured values shown in Fig. 4.

droplet, but it increases dramatically when laser intensity is close to $I_{L, \text { max }}$ (see Fig. 6).

After the laser pulse has traversed the simulation domain the number of rotating electrons and their energy changes slowly for a relatively long time. In Figs. 4(c) and 4(d) the spatial velocity distribution clearly shows that the most energetic electrons rotate inside the expanded droplet at a radius close to the original target radius. This is due to the internal structure of the radial electric field, which has a peak value close to $r=R$ and it is a linear function of radius for $r<R$. This is a consequence of the uniform ion density inside the droplet, while it is quickly decreasing in the plasma corona.

The circular path of the electrons is defined by the balance between the attracting radial electrostatic force and the inertial centrifugal force. In the vacuum region the following equation holds: $\gamma m_{e} v_{\theta}^{2} / r_{e}=E_{r}^{\text {out }}=e Q_{*} /\left(4 \pi \epsilon_{0} r_{e}^{2}\right)$, where $v_{\theta}=c\left(1-1 / \gamma^{2}\right)^{1 / 2}$ is their azimuthal velocity. Here we consider the equilibrium state, where the radial and axial velocity components are negligible. Inside of the droplet the balance equation changes, because the electrostatic force has a different form: $E_{r}^{\text {in }}=e Q_{*} r_{e} /\left(4 \pi \epsilon_{0} R^{3}\right)$. By solving the dimensionless equation $\rho_{e}^{\text {in }}=\rho_{0}$ or $\rho_{e}^{\text {out }}=$ $\rho_{0}$ we obtain the electron energy which is present near the initial surface of the droplet: $\gamma_{R}=\left\{a_{0} \rho_{0}\left(1-e^{-\tau_{L} / 4}\right)+\right.$
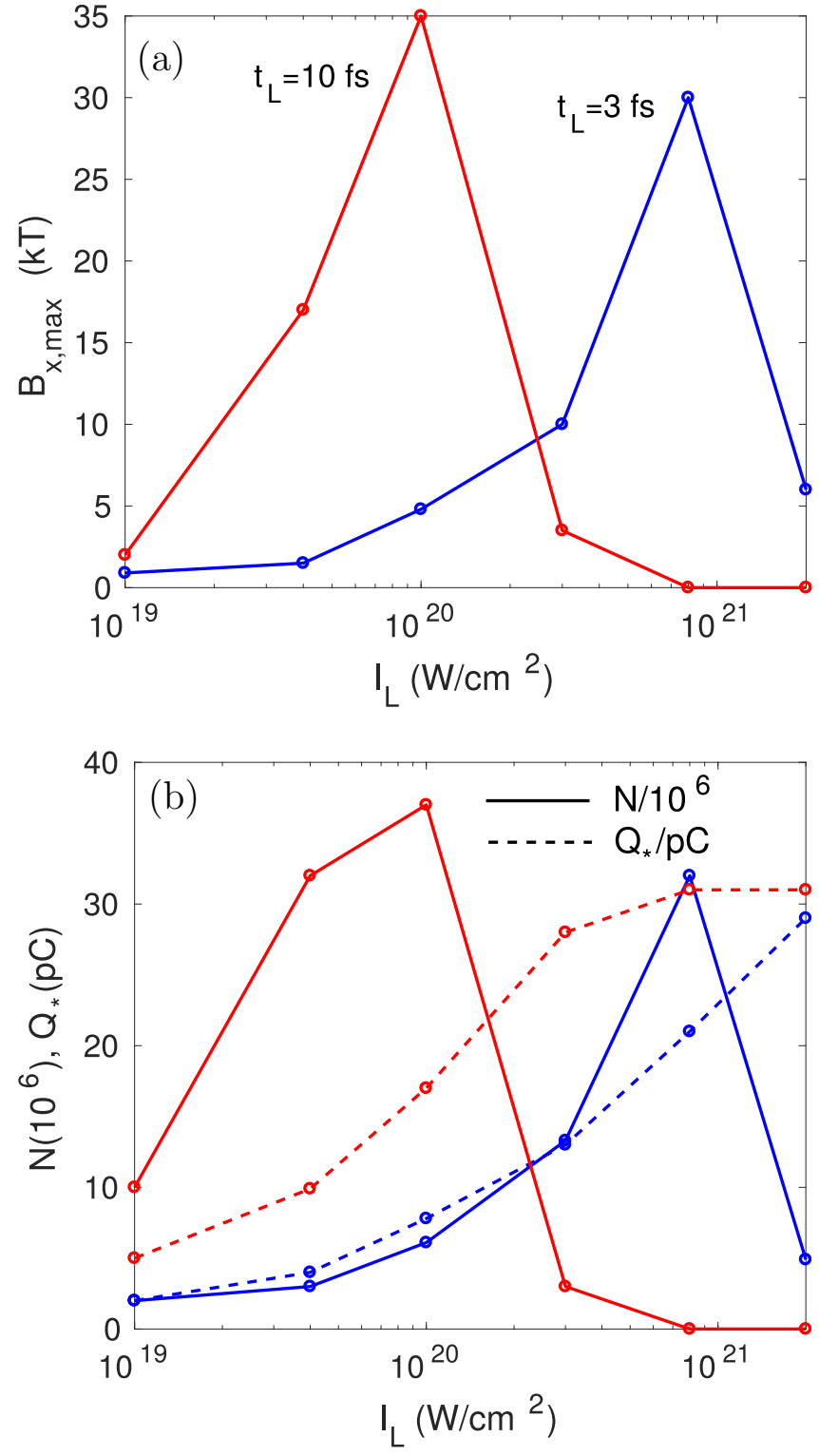

FIG. 6. (left) Peak values of the magnetic field for different laser pulse parameters at $5 \mathrm{fs}$ after the end of the laser pulse. (right) The number of rotating electrons ( $N$, full lines) and the charge of the droplet after interaction $\left(Q_{*}\right.$, dashed lines) for the same simulation parameters.

$\left.\left[4+a_{0}^{2} \rho_{0}^{2}\left(1-e^{-\tau_{L} / 4}\right)^{2}\right]^{1 / 2}\right\} / 2$, where $\tau=\omega_{L} t, \rho=2 \pi r / \lambda_{L}$. We found that the average velocity of electrons taking part in the toroidal current is well approximated by $\bar{v}_{\theta}=v_{\theta R} / 2$, where $v_{\theta R}$ is calculated from $\gamma_{R}$. Starting from the definition $B=\mu_{0} I_{\theta} /(2 r)$ and using an approximate expression for the current $I_{\theta}=N e \bar{v}_{\theta} /(2 \pi R)$ the magnetic field can be expressed in a simplified form:

$$
B_{x}=N B_{0} \frac{\rho_{0}}{\rho} \frac{\bar{v}_{\theta}}{c},
$$

where $B_{0}=\mu_{0} e c /\left(4 \pi R^{2}\right)$. The energy dissipation of electrons via collisional effects can be neglected [30] because the energy transfer from electrons to ions happens on a picosecond timescale, thus the total current is conserved. 
For our simulation parameters one obtains $\bar{v}_{\theta} \approx 0.3 c$, which agrees well with Fig. 4. The maximum magnetic field can be achieved when the laser intensity is close to the critical one [derived from Eq. (1)], when $N \sim 10^{8}$ electrons contribute to $I_{\theta}$, resulting in tens of kT peak value.

The temporal evolution of the generated magnetic field depends only on the radial expansion of the droplet, which is governed by the static electric self-field, described by the following equation:

$$
\frac{d^{2} r}{d t^{2}}=\frac{Z e Q(t)}{4 \pi \epsilon_{0} r^{2} m_{i}},
$$

where the time-dependent charge [Eq. (3)] is used up to the time instance $t_{L}$ and $Q_{*}=Q\left(t_{L}\right)$ for later times. After using the normalization of physical quantities we arrive at a dimensionless equation:

$$
\frac{d^{2} \rho}{d \tau^{2}}=Z a_{0} \frac{m_{e}}{m_{i}} \frac{\rho_{0}^{2}}{\rho^{2}}\left(1-e^{-\tau / 4}\right) .
$$

The solution of Eq. (6) is shown in Fig. 5(a) together with the simulation results. One can see that, at higher intensity, the expansion is faster because $Q_{*}$ is larger, and the magnetic field decays more quickly. This time dependence of the radius will be used in the definition of the magnetic field [Eq. (4)].

The comparison of measured magnetic field with Eq. (4) is presented in Fig. 5(b) for the same simulation parameters as in Fig. 5(a). The only fitting parameter is $N$, which is an increasing function of time during the laser-target interaction. It can be seen that while the laser pulse is interacting with the droplet the model does not fit the simulation because the parameter $N$ is unknown and due to the influence of the laser field. However, one can find a value for $N$ providing the same time dependence as in the simulation and it is very close to the measured quantities presented in Fig. 4. It is worth mentioning that the number of rotating electrons after the interaction is proportional to the laser pulse length as long as $Q_{*}<Q_{m}$.

\section{B. Maximizing the magnetic-field amplitude}

From the analytical theory it is apparent that the magneticfield strength can be enhanced most efficiently by increasing the number of rotating electrons around the droplet $(N)$. This can be done by increasing the pulse length, but the laser field can be effective only if the droplet charge is below $Q_{m}$. Therefore, $Q_{m}$ has to be increased as much as possible by increasing the laser intensity. A maximum laser intensity can be defined at the condition $Q_{m}=e n_{e} 4 \pi R^{3} / 3$, which means that all electrons are removed from the target. The optimum laser intensity is, of course, below this threshold because if all electrons are removed, then the azimuthal current is zero. A large amount of electrons should remain near the droplet and perform a circular motion.

More simulations have been performed at higher laser intensity, where we assume +40 charge state of the ions. At this charge state the threshold laser intensity for complete electron removal in one laser cycle is $4 \times 10^{21} \mathrm{~W} / \mathrm{cm}^{2}$, thus the optimum should be below this value. Figure 6(a) shows that the optimum laser intensity gets lower by increasing the pulse duration. This happens because the longer pulse with
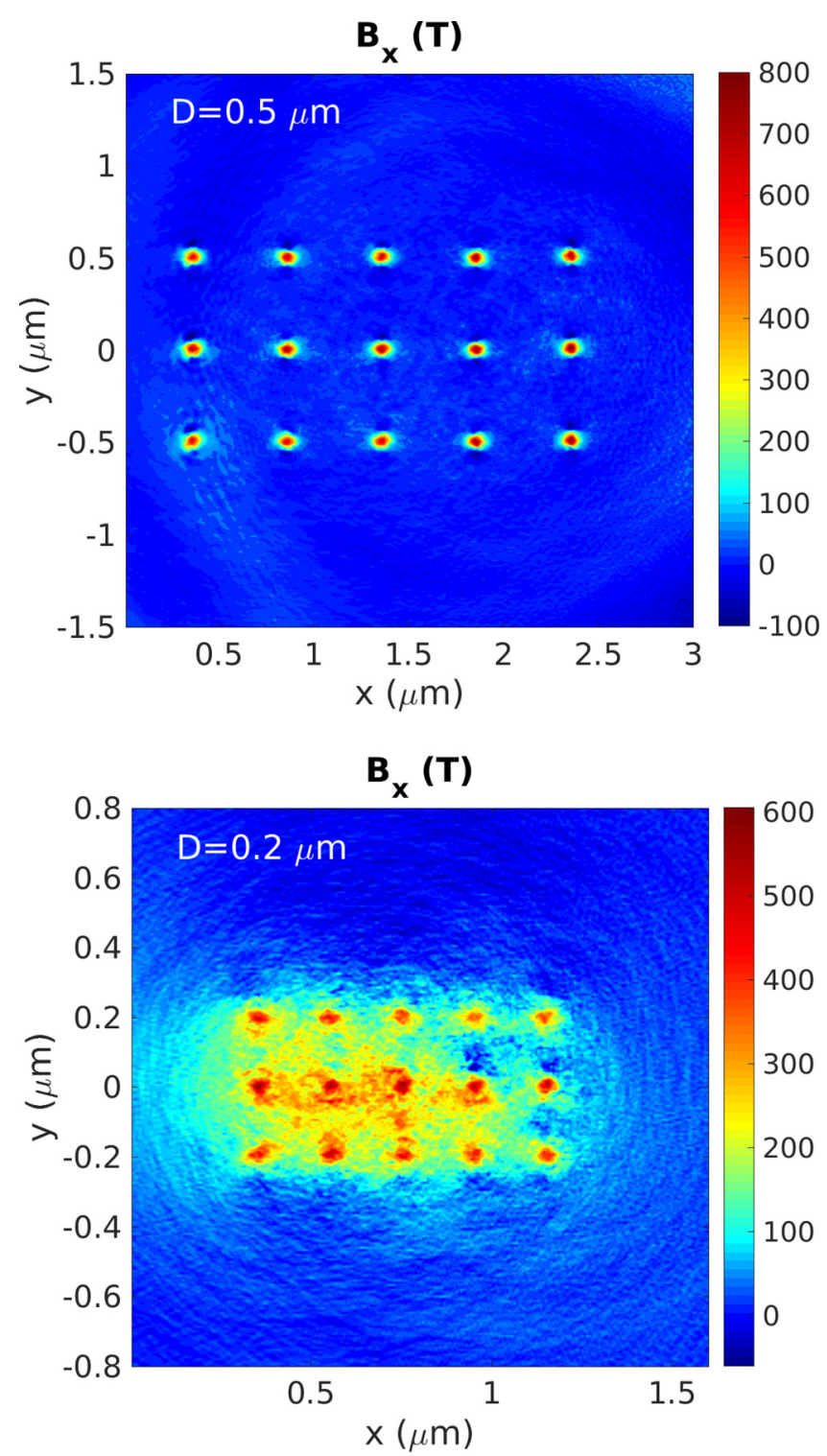

FIG. 7. Magnetic-field distribution in the $x y$ plane $(z=0)$ in the case of uniformly placed droplets with two different separation distances. The peak intensity is $I_{L}=4 \times 10^{18} \mathrm{~W} / \mathrm{cm}^{2}$ and $t_{L}=3 \mathrm{fs}$.

the same intensity as the short pulse removes more electrons and fewer remain in the target to contribute to the azimuthal current. This can be seen in Fig. 6(b), where $Q_{*}$ increases faster by increasing the intensity in the case of longer pulse. As the droplet charge approaches $Q_{m}=31 \mathrm{pC}$ the number of rotating electrons drops suddenly and it happens at lower intensity with a longer pulse. On the other hand, with a fewcycle pulse the optimal intensity is higher than in the case of the longer pulse because the extracted electrons are not pushed far from the target and after the short interaction many electrons are attracted back by the positive droplet.

\section{Interaction with several nano-droplets}

In experiments the longitudinal and transversal dimensions of the focused laser pulse are a few microns, which means that, in the focal volume, the laser interacts with 10 to 20 clusters if between them $\sim 1 \mu \mathrm{m}$ distance is assumed. Since 
the electrons are orbiting very close to the droplet surface the individual magnetic dipoles can be considered independent and do not interact. This is confirmed in Fig. 7 in the case of $D=0.5 \mu \mathrm{m}$, where the parameters are the same as in Fig. 1. In this case the spatially averaged (positive) magnetic field is on the order of $10 \mathrm{~T}$. When the distance between droplets is reduced to $D=0.2 \mu \mathrm{m}$ the positive magnetic fields are merged and a uniform region is obtained where the field strength is about half of the peak value. The average magnetic field is higher in this case, $\left\langle B_{x}\right\rangle \approx 150 \mathrm{~T}$. This regime is similar to the inverse Faraday rotation, where the magnetic field is generated in the whole volume of the laser pulse, but here this magnetic field decays much slower due to the slow motion of positively charged droplets. The quasi-uniform magnetic field also suggests that some high -energy electrons can move around several droplets on a longer trajectory generating a wider coil-like structure.

The average magnetic field surrounding the steady nanodroplets can be compared with the field generated by the same laser pulse in uniform underdense plasma. Such a simulation has been performed, which showed an average field value around 60 T. We can also use Eq. (2.3) from Ref. [31] to estimate the magnetic field at the center of the laser pulse $(r=0)$ :

$$
B_{x, I F R}=\frac{\lambda_{L} e I_{L}}{4 m_{e} \epsilon_{0} \omega^{3} R_{L}^{2}} .
$$

We assume a tightly focused laser pulse, where $\lambda_{L} \approx R_{L} \approx$ $1 \mu \mathrm{m}$. The estimated magnetic field from Eq. (7) is $B_{x, I F R} \approx$ $50 \mathrm{~T}$, which is very close to our simulation results. This indicates that our method is capable of generating the same average magnetic field (or higher) in the laser focal volume as in the case of inverse Faraday rotation, but locally the peak values are 10 or even 100 times higher. The generated magnetic-field amplitude depends mostly on the laser pulse length if the laser intensity is much lower than $I_{L, \max }$. In this low-intensity regime $Q_{*}$ is relatively small and the droplet expansion is slow.
Even if the strong magnetic field exists for more than $100 \mathrm{fs}$ its measurement is really challenging due to the small size it occupies. However, there is an indirect way to detect such high magnetic fields by the detection of fine spectral line structure generated by Zeeman effect. A field of $1 \mathrm{kT}$ is capable of splitting spectral emission lines with $1 \mathrm{eV}$ separation and this gap can be larger than the thermal (Doppler) broadening caused by the heating of ions. Usually the energy transfer from electrons to ions via collisions happens on a picosecond timescale, thus in our case the ions can be considered very cold. If the ion temperature remains below $10 \mathrm{eV}$, then the Doppler broadening is smaller than $0.01 \%$. In the case of $100 \mathrm{eV}$ emitted photon energy it means $<10 \mathrm{meV}$ (millielectronvolt) broadening.

\section{CONCLUSIONS}

We have presented an efficient method for generating large amplitude $(\sim k T)$ magnetic field in clusterized targets. The required laser intensity is slightly above the relativistic threshold and a pulse duration not longer than few laser periods is sufficient. In contrast with the inverse Faraday effect the peak magnetic field is higher and is localized in space, at the position of the clusters. The lifetime of magnetic-dipole moment is defined by the expansion time of the droplet, which can be on the order of hundreds of femtoseconds if heavy droplets are considered, i.e., if the clusterized gas consists of high- $Z$ material, or low-intensity laser pulses are used.

\section{ACKNOWLEDGMENTS}

We acknowledge KIFÜ/NIIF for awarding us access to HPC resource based in Debrecen, Hungary. We also thank to the IT Support at ELI-ALPS for providing computational resources on the internal HPC. The ELI-ALPS project (GINOP2.3.6-15-2015-00001) is supported by the European Union and co-financed by the European Regional Development Fund.
[1] K. Heyde, P. von Neumann-Cosel, and A. Richter, Rev. Mod. Phys. 82, 2365 (2010).

[2] C. P. Koch, M. Lemeshko, and D. Sugny, Rev. Mod. Phys. 91, 035005 (2019).

[3] M. F. Picardi, M. Neugebauer, J. S. Eismann, G. Leuchs, P. Banzer, F. J. Rodríguez-Fortuño, and A. V. Zayats, Light: Sci. Appl. 8, 52 (2019).

[4] J.-Y. Ge, V. N. Gladilin, J. Tempere, J. T. Devreese, and V. V. Moshchalkov, Nat. Commun. 9, 2576 (2018).

[5] J. Wang, H. Dong, and S.-W. Li, Phys. Rev. A 97, 013819 (2018).

[6] M. E. Fisher, Phys. Rev. 113, 969 (1959).

[7] G. Carneiro, Phys. Rev. B 72, 144514 (2005).

[8] R. B. Griffiths, Phys. Rev. 176, 655 (1968).

[9] T. Blasberg and D. Suter, Phys. Rev. Lett. 69, 2507 (1992).

[10] R. Schwengner, S. Frauendorf, and A. C. Larsen, Phys. Rev. Lett. 111, 232504 (2013).

[11] N. Itoh, Phys. Rev. A 43, 1002 (1991).
[12] J. R. Sims, D. G. Rickel, C. A. Swenson, J. B. Schillig, G. W. Ellis, and C. N. Ammerman, IEEE Trans. Appl. Supercond. 18, 587 (2008).

[13] B. A. Remington, R. P. Drake, and D. D. Ryutov, Rev. Mod. Phys. 78, 755 (2006).

[14] V. Kaymak, A. Pukhov, V. N. Shlyaptsev, and J. J. Rocca, Phys. Rev. Lett. 117, 035004 (2016).

[15] Z. Lécz and A. Andreev, New J. Phys. 20, 033010 (2018).

[16] M. Borghesi, A. J. MacKinnon, A. R. Bell, R. Gaillard, and O. Willi, Phys. Rev. Lett. 81, 112 (1998).

[17] M. Tatarakis, I. Watts, F. N. Beg, E. L. Clark, A. E. Dangor, A. Gopal, M. G. Haines, P. A. Norreys, U. Wagner, M.-S. Wei, M. Zepf, and K. Krushelnick, Nature (London) 415, 280 (2002).

[18] G. Sarri, A. Macchi, C. A. Cecchetti, S. Kar, T. V. Liseykina, X. H. Yang, M. E. Dieckmann, J. Fuchs, M. Galimberti, L. A. Gizzi, R. Jung, I. Kourakis, J. Osterholz, F. Pegoraro, A. P. L. Robinson, L. Romagnani, O. Willi, and M. Borghesi, Phys. Rev. Lett. 109, 205002 (2012). 
[19] L. G. Huang, H. Takabe, and T. E. Cowan, High Power Laser Sci. Eng. 7, e22 (2019).

[20] J. Deschamps, M. Fitaire, and M. Lagoutte, Phys. Rev. Lett. 25, 1330 (1970).

[21] Z. Najmudin, M. Tatarakis, A. Pukhov, E. L. Clark, R. J. Clarke, A. E. Dangor, J. Faure, V. Malka, D. Neely, M. I. K. Santala, and K. Krushelnick, Phys. Rev. Lett. 87, 215004 (2001).

[22] N. Naseri, V. Y. Bychenkov, and W. Rozmus, Phys. Plasmas 17, 083109 (2010).

[23] Z. Lécz, I. V. Konoplev, A. Seryi, and A. Andreev, Sci. Rep. 6, 36139 (2016).

[24] S. Fujioka, Z. Zhang, K. Ishihara, K. Shigemori, Y. Hironaka, T. Johzaki, A. Sunahara, N. Yamamoto, H. Nakashima, T. Watanabe, H. Shiraga, H. Nishimura, and H. Azechi, Sci. Rep. 3, 1170 (2013).
[25] V. T. Tikhonchuk, M. Bailly-Grandvaux, J. J. Santos, and A. Poyé, Phys. Rev. E 96, 023202 (2017).

[26] T. Fennel, K.-H. Meiwes-Broer, J. Tiggesbäumker, P.-G. Reinhard, P. M. Dinh, and E. Suraud, Rev. Mod. Phys. 82, 1793 (2010).

[27] S. Ter-Avetisyan, M. Schnürer, H. Stiel, U. Vogt, W. Radloff, W. Karpov, W. Sandner, and P. V. Nickles, Phys. Rev. E 64, 036404 (2001).

[28] V. Savinov, N. Papasimakis, D. P. Tsai, and N. I. Zheludev, Comm. Phys. 2, 69 (2019).

[29] M. Lezius, S. Dobosz, D. Normand, and M. Schmidt, Phys. Rev. Lett. 80, 261 (1998).

[30] E. Gamaly and A. Rode, Prog. Quantum Electron. 37, 215 (2013).

[31] A. A. Frolov, Plasma Phys. Rep. 30, 698 (2004). 